\title{
O Impacto das Habilidades Sociais para a Depressão em Estudantes Universitários
}

\author{
Alessandra Turini Bolsoni-Silva ${ }^{1}$ \\ Universidade Estadual Paulista \\ Sonia Regina Loureiro \\ Universidade de São Paulo
}

\begin{abstract}
RESUMO - A depressão é prevalente em universitários, embora se verifique escassez de estudos sobre habilidades sociais associadas à depressão. Objetivou-se comparar as habilidades sociais e as percepções de consequências nas interações de universitários com depressão ( $\mathrm{n}=64$ ) em relação a um grupo não clínico ( $\mathrm{n}=64)$; e verificar o valor preditivo das habilidades sociais. Habilidades sociais e saúde mental foram mensuradas por instrumentos aferidos. Constatou-se diferenças significativas em comunicação, afeto, expressar sentimentos negativos, lidar com críticas e falar em público. O grupo com depressão relatou mais consequências e sentimentos negativos nas interações sociais. Na análise de regressão identificou-se que um repertório deficitário de habilidades sociais foi preditor de depressão, o que sugere a relevância de programas de prevenção e intervenção para essa população.
\end{abstract}

Palavras chave: habilidades sociais, universitários, saúde mental, depressão, prevenção

\section{The Impact of Social Skills on Depression of University Students}

\begin{abstract}
Depression is prevalent in university students, and there is a gap in relation to studies about social skills associated to depression. The objective of this study was to compare the social skills and the perceptions of consequences in interactions of students with depression ( $n=64)$, compared to a non-clinical group ( $n=64)$, and verify the predictive value of social skills. Social skills and mental health were measured by validated instruments. Significant differences in communication, affection, expressing negative feelings, dealing with criticism and public speaking were found. The group with depression reported more negative consequences and feelings in social interactions. Regression analysis identified that a deficient repertoire of social skills was predictor of depression, what suggests the relevance of prevention and intervention programs for this population.
\end{abstract}

Keywords: social skills, university students, mental health, depression, prevention

A depressão é um dos transtornos mais prevalentes na população em geral (Andrade et al. 2012; Marrochín, 2011; Richards, 2011) e também na universitária (Fang, Young, Golshan, Moutier, \& Zisook, 2010; Neves \& Dalgalarrondo, 2007), trazendo implicações para o funcionamento social, relacionamento interpessoal, alta taxa de morbidade e de mortalidade (Razzouk, Alvarez, \& Mari, 2009; Richards, 2011), correspondendo a $70 \%$ dos casos de suicídios no mundo, sobretudo em jovens (Razzouk, Alvarez \& Mari, 2009). O risco para as mulheres é duas ou três vezes maior que para os homens, seja na ocorrência, seja na gravidade (Andrade et al. 2012), sobretudo quando da exposição precoce a adversidades, tais como violência, morte parental e divórcio (Dozois \& Dobson, 2004). A presença de comorbidades ocorre na metade dos casos, tendo a probabilidade de $50 \%$ para ansiedade (Johanson, Carlbring, Heedman, Paxling, \& Andersson, 2013; Starr, Hammem, Connolly, \& Brennan, 2014), sendo esses casos mais graves, com maiores comprometimentos físicos e com piores prognósticos (Newby et al., 2013). O termo depressão descreve um sintoma, um estado emocional e uma síndrome clínica (Morrison-Valfre, 2009). Segundo o DSM-V (2014), os transtornos depressivos se caracterizam pela presença de humor triste, vazio ou

1 Endereço para correspondência: Faculdade de Ciências - UNESP, Campus de Bauru, Faculdade de Ciências, Depto de Psicologia, Av. Eng. Luiz Edmundo Carrijo Coube, 14-01, Vargem Limpa. Bauru, SP, Brasil. CEP: 17.033-360.E-mail: bolsoni@fc.unesp.br irritável, acompanhado de alterações somáticas e cognitivas que afetam a capacidade de funcionamento (American Psychological Association [APA], 2014). A prevalência de depressão em universitários tende a ser maior que na população geral. Para estudantes universitários brasileiros a prevalência da depressão foi verificada para alunos de medicina em 40,5\% (Costa et al.,2012), com estudantes da Malásia, a taxa de depressão moderada foi de $27,5 \%$ (Shamsuddin et al.,2012) e, com universitários do Peru, a taxa de depressão foi de 34,1\% (Osada,2010).

$\mathrm{Na}$ vida universitária, muitos estressores se fazem presentes configurando-se como fatores de risco para a depressão, dentre esses se destacam: (a) déficits de habilidades sociais (Dozois \& Dobson, 2004); (b) lidar com interações sociais aversivas (Dozois \& Dobson, 2004); (c) estressores ambientais (Morrison-Valfre, 2009); (d) experiências negativas da vida adulta e circunstâncias sociais (Morrison-Valfre, 2009). Por outro lado, são considerados fatores de proteção para a depressão: ter altas habilidades de inteligência, de resolver problemas e de enfrentamento, boa competência acadêmica, e ter pais com boa saúde mental, contar com suporte familiar e tolerar emoções negativas (Dozois \& Dobson, 2004). Dados os objetivos do estudo, abordar-se-ão as associações entre depressão e habilidades sociais.

A definição de habilidades sociais tem sido objeto de variada apresentação entre os autores da área. Libet 
e Lewinsohn (1973), desde a década de 70, definiam as habilidades sociais em termos das consequências sociais dos comportamentos, chamando a atenção para as sequências de comportamentos enquanto ações emitidas pelo indivíduo e para as reações eliciadas em seu ambiente. Bolsoni-Silva e Carrara (2010) definem as habilidades sociais como um conjunto de comportamentos operantes que parecem aumentar a probabilidade de se obter reforçador positivo frente às demandas interpessoais e minimizam as perdas nas interações sociais. Del Prette e Del Prette (2013) ressaltam que as habilidades sociais se referem a diferentes classes de comportamentos sociais do repertório do indivíduo para lidar com as situações interpessoais, enquanto a competência social remete aos efeitos do desempenho das habilidades no contexto de vida da pessoa. Considerando definições de habilidades sociais, é importante avaliar o repertório de habilidades sociais e as consequências que produzem no ambiente social. Nesse sentido, os comportamentos depressivos afetam o recebimento de suporte e o relacionamento entre esse suporte social e a depressão tem se mostrado bidirecional (Marroquín, 2011).

De acordo com Segrin (2000), com base em uma revisão da literatura, a combinação entre déficits de habilidades sociais e eventos negativos da vida são condições favorecedoras de depressão. Enquanto uma pessoa com bom repertório de habilidades pode identificar o tipo e quantidade de suporte social que será efetivo para lidar com os eventos estressantes, as pessoas com tais déficits experienciam mais estressores e têm menos assistência e suporte social para lidar com esses eventos quando eles ocorrem. $\mathrm{O}$ autor ressalta ainda que déficits em habilidades sociais podem se associar a depressão e, em alguns casos, tais dificuldades podem também ser um sintoma de depressão.

Edmondson, Conger e Conger (2007) verificaram que, quanto maior o nervosismo e a tristeza, menor os escores de competência social e expressividade afetiva. Na mesma direção, Fitts, Sebby e Zlokovich (2009) examinaram o estilo de humor de 132 alunos universitários americanos, constatando que a afiliação, auto-reforço (enhancing), comportamento agressivo e autodestrutivo e a percepção de competência social estavam relacionados com timidez e solidão.

Tais achados também têm respaldo em pesquisas recentes. Evraire e Dozois (2011) constataram que pessoas com depressão emitem alguns comportamentos interpessoais, como buscar excessivamente reafirmação e buscar feedback negativo, os quais trazem consequências negativas e, nesses momentos, a pessoa busca tranquilidade com pessoas próximas, na tentativa de aumentar o efeito positivo e aliviar sua insegurança, porém nem sempre de maneira satisfatória. Biasoto (2013), em estudo conduzido com universitários brasileiros sobre habilidades sociais e neuroticismo, constatou que a depressão se correlacionou com menor repertório social. Bolsoni-Silva e Guerra (2014), com estudantes universitários brasileiros, verificaram que aqueles que apresentavam depressão tinham menor repertório de habilidades sociais, referindo sentirem-se mal com maior frequência frente as interações, independente de quais fossem os interlocutores. Aradilla-Herrero, Tomás-Sábado e Gómez-Benito (2014), em estudo conduzido com estudantes de enfermagem constataram que a depressão foi preditor significativo de suicídio e de baixa autoestima. Bolsoni-Silva e Loureiro (2015) constataram em estudo com universitários brasileiros que a depressão foi preditora de menor repertório geral de habilidades.

Verifica-se assim, que déficit de habilidades sociais, ainda que não seja o único fator de risco para depressão, é importante de ser avaliado, pois pode dificultar a resolução de problemas e o enfrentamento dos desafios da vida universitária. Os estudos que descrevem repertórios de habilidades sociais em universitários, ora destacam a relevância da comunicação, ora da resolução de problemas, mas poucos se ocupam em avaliar as consequências produzidas nas interações sociais, comparando grupos clínico e não clínico.

O presente estudo se insere nessa lacuna objetivando: (a) comparar os indicadores comportamentais de habilidades sociais e as percepções de consequências nas interações por parte de universitários com depressão em relação a um grupo não clínico; (b) identificar indicadores de habilidades sociais que têm valor preditivo para a depressão.

\section{Método}

\section{Aspectos Éticos}

Este trabalho é parte de um projeto que prevê avaliação e intervenção junto a universitários cujo título é Análise das habilidades sociais de grupos universitários, o qual foi aprovado em 30 de agosto de 2007 pelo Comitê de Ética em Pesquisa da universidade em que está vinculado (Processo $\left.\mathrm{n}^{\mathrm{o}} 1315 / 46 / 01 / 07\right)$.

\section{Participantes}

Participaram deste estudo 128 estudantes universitários, graduandos de diferentes cursos de uma universidade pública do interior do estado de São Paulo- Brasil, distribuídos em todos os anos dos cursos que frequentavam, sendo 64 clínicos para depressão e 64 não clínicos. Dados demográficos foram comparados estatisticamente (Teste Crosstabs) não sendo identificadas diferenças significativas entre os grupos clínico para depressão e não clínico para período de estudo (integral $\mathrm{x}$ noturno), estado civil (solteiro, casado), namoro, tipo de moradia (sozinho, república, família, namorado), tipo de curso (áreas humanas, biológicas, exatas), sexo, trabalho e idade.

\section{Instrumentos}

Questionário de Avaliação de Habilidades Sociais e Contextos para Universitários - QHC-Universitários. É um questionário composto por questões que se referem à forma como o participante se comporta com relação a seus pais, amigos, namorado (a), entre outros, bem como os contextos em que ocorrem os comportamentos, como os interlocutores reagem a eles e os sentimentos dos 
universitários nessas ocasiões. O instrumento final ficou constituído pelos seguintes tópicos: (a) Comunicação, (b) Expressividade (Sentimentos positivos, negativos e opiniões), (c) Críticas (fazer e receber críticas),(d) Falar em público (falar para público - conhecido e desconhecido, frequência e dificuldade em apresentar seminários) e (e) Informações Adicionais (caso a pessoa deseje acrescentar algo que não foi abordado no questionário). Foi validado por Bolsoni-Silva (2011) com satisfatórios resultados de validade discriminativa, concorrente e de critério.

Inventário de Habilidades Sociais- IHS-Del-Prette (Del Prette \& Del Prette, 2001). É um instrumento composto de 38 itens, que tem como objetivo avaliar dimensões situacionais e comportamentais das habilidades sociais de auto-relato. Esses itens são agrupados em cinco fatores amplos: Fator 1 - Enfrentamento e auto-afirmação com risco, Fator 2 - Auto-afirmação na expressão de sentimento positivo, Fator 3 - Conversação e desenvoltura social, Fator 4 - Auto-exposição a desconhecidos e situações novas e Fator 5 - Autocontrole da agressividade. O IHS-Del Prette possui adequação quanto às qualidades psicométricas de validade concomitante e de sua fidedignidade ou estabilidade temporal.

Inventário de Depressão de Beck (BDI). Foi incluído no estudo como uma medida de saúde mental, enquanto um rastreador de indicadores de depressão. O instrumento foi traduzido, adaptado e validado para a população brasileira por Cunha (2001), é auto-administrado, composto por 21 itens que avaliam a gravidade dos sintomas de depressão, os quais são pontuados em uma escala likert de 0 (absolutamente não) a 3 (gravemente), permite classificações da depressão nos níveis mínimo (0-11), leve (12-19), moderado (20-35) e grave (36-63). Originalmente criado para uso com pacientes psiquiátricos, esse instrumento também se mostrou adequado para o uso na população geral.

Entrevista Clínica Estruturada para o DSM-IV (SCID-I - versão clínica - Del-Ben et al., 2001). Foi incluída no estudo por ser considerada uma medida de saúde mental que permite a classificação diagnóstica por transtornos, assim como a classificação de ausência de transtorno psiquiátrico, tratando-se de um instrumento diagnóstico, considerado padrão ouro em estudos clínicos. Proposta por First et al. (1997), traduzida e adaptada para o português por Del-Ben et al. (2001) também possui satisfatórias propriedades psicométricas. Utilizou-se esse instrumento baseado no DSM-IV, pois a vigência DSM-V é recente e a versão correspondente da SCID ainda está sendo aferida.

\section{Procedimentos de Coleta de Dados}

A coleta dos dados foi coletiva, após autorização das Coordenações de Cursos e dos respectivos professores que foram contatados em salas de aula, momentos em que foram oferecidos os devidos esclarecimentos sobre os objetivos da mesma. Os participantes que concordaram participar da pesquisa assinaram um Termo de Consentimento Livre e Esclarecido e receberam uma carta explicativa sobre os objetivos do projeto e um caderno contendo instruções sobre a aplicação dos instrumentos acima descritos, com exceção da SCID, que foi conduzida por telefone posteriormente. A amostra total foi constituída por 1346, dos quais 609 universitários também foram entrevistados por telefone para a aplicação da SCID. Para a presente pesquisa foram selecionados os participantes que apresentavam indicadores de depressão pelo BDI ou diagnóstico de depressão pela SCID.

O Grupo de Depressão foi composto, então, por 64 participantes ( 24 homens e 40 mulheres). Desses universitários, 35 responderam tanto o BDI quanto a SCID e 29 responderam apenas ao BDI. Dos universitários que responderam aos dois instrumentos, 11 preencheram critério para depressão apenas na SCID depressão e apenas dois deles, em ambos os instrumentos (SCID e BDI). Quanto à distribuição por cursos têm-se: 24 universitários da área de exatas, 37 da área de humanas e três de biológicas. Do grupo não clínico, composto por 413 universitários, que não pontuavam em qualquer dos instrumentos de saúde mental utilizados, foram selecionados 64 pareados com as características do grupo clínico quanto ao tipo de curso e sexo, ou seja, no grupo não clínico foi incluído o mesmo número de universitários do sexo feminino e masculino, bem como o mesmo número de participantes que compôs o grupo clínico. Nota-se que foi possível parear 61 participantes não clínicos considerando ambas as variáveis (sexo e curso), os demais três participantes foram pareados apenas quanto a sexo, de forma que a amostra ficou constituída por 24 homens clínicos e 24 homens não clínicos e por 40 mulheres clínicas e 40 não clínicas. As variáveis sexo e curso foram controladas na composição dos grupos e as demais foram tratadas estatisticamente, sem diferenças entre os mesmos, garantindo amplo controle de variáveis.

\section{Procedimentos de Tratamento e Análise de Dados}

Para compor o grupo clínico foram considerados indicadores de depressão (BDI) com ou sem confirmação diagnóstica (SCID), com ou sem outras comorbidades. A variável dependente do estudo é a depressão, que compôs o banco de dados de maneira binária, presença ou ausência. O grupo clínico para depressão foi comparado com um grupo não clínico utilizando o teste $t$. Os resultados estão organizados na forma de tabelas, considerando significância de $p$ menor ou igual a 0,05 . Os grupos clínico para depressão e não clínico foram comparados estatisticamente (Teste $t$ de Student) quanto às categorias previstas pelos dois instrumentos de habilidades sociais (QHC-Universitários e IHS-Del Prette). As variáveis com diferenças estatísticas no Teste $t$ de Student foram incluídas no modelo de Regressão Logística Binária Múltipla, de modo a verificar o peso delas para a depressão, considerando os cinco fatores do QHCUniversitários e os cinco fatores do IHS-Del Prette, de forma a descrever quais as categorias de habilidades sociais avaliadas possuem valor na predição para a Depressão. As condições para a realização da Regressão Logística Binária foram consideradas, procedeu-se à verificação da multicolinearidade entre estas variáveis, que foi realizada por meio do cálculo do Fator de Inflação da Variância 
(VIF). Entre as variáveis incluídas no modelo de predição, nenhuma excedeu valores de VIF maior que 5,0 podendo ser, portanto, consideradas como variáveis independentes e a adequação do modelo ajustado foi avaliada pelo teste de Hosmer-Lemeshow.

\section{Resultados}

A seção de resultados apresenta as comparações entre os grupos clínico e não clínico para depressão quanto às categorias amplas do QHC-Universitários e IHS-Del Prette (Tabela 1) e quanto a subcategorias do QHC-Universitários (Tabela 2). A Tabela 3 apresenta os resultados da Regressão Logística para os fatores do QHC-Universitários e do IHSDel Prette, considerando a variável ser clínico ou não para depressão.

Conforme a Tabela 1, verificou-se que das categorias de frequência do QHC-Universitários - Parte 1 as categorias manter conversação, apresentar seminários e falar em público e os correspondentes Fator 1 comunicação e afeto e Fator 3 falar em público apresentaram maiores médias para o grupo não clínico na comparação com o clínico para a depressão. Com relação ao QHC-Universitários -
Parte 2, que investiga características dos comportamentos, verificou-se que os grupos se diferenciaram em oito das nove categorias previstas. $\mathrm{Na}$ direção esperada o grupo clínico para depressão teve maiores médias em: comportamento não habilidoso, consequência negativa, sentimento negativo e em Dificuldades. Por outro lado, o grupo não clínico apresentou maiores médias em: Comportamento habilidoso, Conseqüência positiva, Sentimento positivo e Potencialidades. Adicionalmente, todos os Fatores do IHSDel Prette também diferenciaram os grupos, sendo as médias maiores para o grupo não clínico.

Como descrito em Método, o QHC-Universitários - Parte 2 investiga, para cada um dos comportamentos de habilidades sociais, as situações em que ocorrem, as ações do participante (comportamentos habilidosos e não habilidoso), as ações dos interlocutores (consequências positivas, consequências negativas) e os sentimentos do universitário (sentimentos positivos e sentimentos negativos). A Tabela 2 apresenta os resultados das comparações entre os grupos clínico para depressão e não clínico que foram significativas.

Os resultados, expressos na Tabela 2, mostraram que o grupo clínico para depressão apresentou mais dificuldades, conforme esperado. Nesse sentido, o grupo clínico apresentou

Tabela 1. Categorias do QHC-Universitários e do IHS-Del Prette nas comparações dos Grupos Clínico para Depressão

\begin{tabular}{|c|c|c|c|}
\hline $\begin{array}{l}\text { Categorias do QHC- } \\
\text { Universitários }\end{array}$ & $\begin{array}{l}\text { Clínico } \\
\text { Média (D }\end{array}$ & $\begin{array}{l}\text { Não clínico } \\
\text { io Padrão) }\end{array}$ & $t^{*}$ \\
\hline \multicolumn{4}{|c|}{ QHC-Universitários - Freqüências comportamentais - Parte 1} \\
\hline Conversação & $6,25(1,84)$ & $7,23(1,46)$ & $-3,353 * *$ \\
\hline Apresentar seminários & $1,89(1,26)$ & $2,48(1,10)$ & $-2,840 * *$ \\
\hline Falar em público & $2,11(1,35)$ & $2,61(1,16)$ & $-2,248^{*}$ \\
\hline Fator 1-Comunicação e afeto & $17,66(5,30)$ & $19,53(5,11)$ & $-2,036^{*}$ \\
\hline Fator 3-Falar em público & $4,00(2,21)$ & $5,01(1,95)$ & $-2,968 * *$ \\
\hline \multicolumn{4}{|c|}{ QHC-Universitários - Características comportamentais - Parte 2} \\
\hline Comportamento habilidoso & $13,25(5,53)$ & $15,73(4,71)$ & $-2,737 * *$ \\
\hline Comportamento não habilidoso & $7,73(4,53)$ & $5,33(3,66)$ & $3,304 * *$ \\
\hline Consequência positiva & $20,70(8,52)$ & $25,77(6,45)$ & $-3,789 * *$ \\
\hline Consequência negativa & $5,11(4,07)$ & $2,22(2,18)$ & $5,008 * *$ \\
\hline Sentimento positivo & $22,86(15,41)$ & $31,97(12,80)$ & $-3,638 * *$ \\
\hline Sentimento negativo & $23,53(19,05)$ & $10,05(9,09)$ & $5,110 * *$ \\
\hline Potencialidades & $77,63(33,67)$ & $95,78(25,34)$ & $-3,447 * *$ \\
\hline Dificuldades & $36,38(25,08)$ & $17,59(12,34)$ & $5,375^{* *}$ \\
\hline \multicolumn{4}{|c|}{ IHS-DEL-PRETTE } \\
\hline Fator 1 & $19,05(7,21)$ & $23,83(7,50)$ & $-3,676^{* *}$ \\
\hline Fator 2 & $17,55(5,53)$ & $22,05(3,88)$ & $-5,333 * *$ \\
\hline Fator 3 & $15,55(4,85)$ & $19,22(4,40)$ & $-4,485 * *$ \\
\hline Fator 4 & $8,23(3,52)$ & $10,91(3,17)$ & $-4,517 * *$ \\
\hline Fator 5 & $6,92(2,37)$ & $8,31(2,36)$ & $-3,332 * *$ \\
\hline Outros & $15,45(4,05)$ & $17,55(3,31)$ & $-3,202 * *$ \\
\hline Total & $82,41(20,77)$ & $101,38(15,20)$ & $-5,896 * *$ \\
\hline
\end{tabular}


maiores médias em comportamento não habilidoso (receber crítica, apresentar seminários e falar em público), consequência negativa (conversar, expressar sentimento positivo, expressar sentimento negativo, expressar opinião, fazer crítica, apresentar seminários, falar em público) e sentimento negativo (conversar, expressar sentimento positivo, expressar sentimento negativo, expressar opiniões, fazer críticas, receber críticas, apresentar seminários e falar em público).
Em direção inversa, o grupo não clínico teve maiores médias em comportamento habilidoso (expressar sentimento positivo, expressar sentimento negativo, apresenta seminários), consequência positiva (conversar, expressar sentimento positivo, apresenta seminários) e sentimentos positivos (conversar, expressar sentimentos positivos, falar em público). O grupo não clínico também relatou mais frequentemente conversar em maior número de situações na comparação com o clínico.

Tabela 2. Subcategorias do QHC-Universitários - Parte 2 na comparação entre os Grupos Clínico para Depressão e Não Clínico. Expressos os resultados das comparações com diferenças estatisticamente significantes

\begin{tabular}{|c|c|c|c|}
\hline \multirow{2}{*}{$\begin{array}{l}\text { QHC-Universitários - Características } \\
\text { comportamentais: Subcategorias }\end{array}$} & Clínico & Não clínico & \multirow{2}{*}{$t^{* *}$} \\
\hline & \multicolumn{2}{|c|}{ Média (Desvio Padrão) } & \\
\hline Conversação: Situações & $5,78(2,43)$ & $7,14(2,56)$ & $-3,085 * *$ \\
\hline Conversação: conseqüências positivas & $6,80(3,48)$ & $8,56(2,88)$ & $-3,126^{* *}$ \\
\hline Conversação: conseqüências negativas & $1,56(1,73)$ & $0,72(1,13)$ & $3,269 * *$ \\
\hline Conversação: sentimentos positivos & $5,77(3,86)$ & $8,69(3,48)$ & $-4,501 * *$ \\
\hline Conversação: sentimentos negativos & $4,72(3,93)$ & $1,50(2,22)$ & $5,702 * *$ \\
\hline Expressa sent. positivos: compto hábil. & $5,55(3,57)$ & $6,73(3,40)$ & $-1,929 *$ \\
\hline Expressa sent. positivos: conseq. positiva & $2,95(1,30)$ & $3,94(0,97)$ & $-4,843 * *$ \\
\hline Expressa sent. positivos: conseq. negativa & $0,86(0,89)$ & $0,38(0,68)$ & $3,466 * *$ \\
\hline Expressa sent. positivos: sent.positivos & $5,88(4,00)$ & $8,83(4,23)$ & $-4,061 * *$ \\
\hline Expressa sent. positivos: sent. negativos & $2,97(3,25)$ & $0,95(1,16)$ & $4,671 * *$ \\
\hline Expressa sent. negativos: compto habil. & $1,00(0,99)$ & $1,11(1,14)$ & $2,028 *$ \\
\hline Expressa sent. negativos: conseq. negativa & $0,92(1,21)$ & $0,39(0,63)$ & $3,107 * *$ \\
\hline Expressa sent. negativos: sent.negativo & $3,53(3,32)$ & $1,39(2,21)$ & $4,292 * *$ \\
\hline Expressa opiniões: conseq. negativas & $0,98(1,18)$ & $0,45(0,75)$ & $3,044 * *$ \\
\hline Expressa opiniões: sentimentos negativos & $2,30(2,87)$ & $1,03(1,54)$ & $3,106^{* *}$ \\
\hline Faz crítica: conseqüências negativas & $0,81(1,08)$ & $0,38(0,68)$ & $2,741 * *$ \\
\hline Faz crítica: sentimentos negativos & $2,69(3,50)$ & $1,31(2,00)$ & $2,729 * *$ \\
\hline Recebe crítica: compto não habilidoso & $0,59(0,87)$ & $0,23(0,68)$ & $2,602 * *$ \\
\hline Recebe crítica: sentimentos negativos & $2,28(3,17)$ & $0,88(1,61)$ & $3,161 * *$ \\
\hline Apresenta seminários: compto habilidoso & $0,36(0,60)$ & $0,63(0,77)$ & $-2,182^{*}$ \\
\hline Apresenta seminários: compto não habil. & $2,34(1,49)$ & $1,75(1,30)$ & $2,402 * *$ \\
\hline Apresenta seminários: conseq.positiva & $1,05(0,68)$ & $1,33(0,54)$ & $-2,606^{* *}$ \\
\hline Apresenta seminários: conseq. negativa & $0,36(0,63)$ & $0,14(0,43)$ & $2,300 * *$ \\
\hline Apresenta seminários: sentimento negativo & $2,53(2,15)$ & $1,50(1,28)$ & $3,299 * *$ \\
\hline Fala em público: compto não habilidoso & $2,08(1,69)$ & $1,47(1,13)$ & $2,397 * *$ \\
\hline Fala em público: conseqüência negativa & $0,42(0,71)$ & $0,14(0,43)$ & $2,713 * *$ \\
\hline Fala em público: sentimento positivo & $1,48(1,69)$ & $2,20(1,73)$ & $-2,378 * *$ \\
\hline Fala em público: sentimento negativo & $2,52(2,26)$ & $1,48(1,49)$ & $3,047 * *$ \\
\hline
\end{tabular}

Nota. Teste $t$ de Student; $p \leq 0,05(*)$ ou $p \leq 0,01(* *)$

Tabela 3. Regressão Logística considerando as categorias de habilidades sociais avaliadas

\begin{tabular}{lllll}
\hline & Sig & $\operatorname{Exp}(\boldsymbol{B})$ & Lower & Upper \\
\hline Potencialidades - QHC - Parte 2 & 0,000 & 1,049 & 1,024 & 1,074 \\
Dificuldades - QHC - Parte 2 & 0,000 & 0,892 & 0,851 & 0,935 \\
Auto-afirmação - sentimento positivo - Fator 2 - IHS-Del Prette & 0,017 & 1,158 & 1,027 & 1,306 \\
Conversação e desenvoltura social -Fator 3 - IHS-Del Prette & 0,000 & 1,132 & 1,011 & 1,269 \\
\hline
\end{tabular}


Conforme a Tabela 3, os indicadores de habilidades sociais que entraram no modelo final para depressão foram: Potencialidades e Dificuldades do QHC-Universitários e Fator 2 - Auto-afirmação na expressão de sentimento positivo e Fator 3 - Conversação e desenvoltura social do IHS-Del Prette.

\section{Discussão}

Os resultados obtidos, de maneira geral, confirmaram a associação de déficts em habilidades sociais e depressão, em concordância com diversos estudos (Biasoto, 2013; BolsoniSilva \& Loureiro, 2015; Bolsoni-Silva \& Guerra, 2014; Edmondson et al. 2007; Fitts et al., 2009; Segrin, 2000). Verificou-se que a frequência e a qualidade de habilidades sociais dos universitários diferenciaram os grupos com e sem depressão, desde comportamentos que aparentemente envolviam menos complexidade, como conversar e expressar afeto, a comportamentos que exigiam maior assertividade, como no caso de expressar sentimentos negativos, opiniões e lidar com críticas, com sentido semelhante aos relatados por Bolsoni-Silva e Guerra (2014). Destaca-se que os comportamentos de falar em público, sobretudo o de apresentar seminários diferenciou os grupos clínico e não clínico, diferentemente do estudo de Bolsoni-Silva e Loureiro (2015) que identificaram tais déficits em grupos de universitários ansiosos, mas não para os deprimidos. Tal diferença pode ser resultado do tipo de delineamento, na presente pesquisa sexo e escolaridade foram controladas o que não ocorreu em Bolsoni-Silva e Loureiro (2015).

O grupo não clínico, portanto, apresentou maiores médias na frequência e na qualidade das habilidades sociais, além de percepção mais positiva das consequências produzidas nas interações sociais (consequências positivas, sentimentos positivos e potencialidades). Tais achados avançam em relação a outros estudos na área (Biasoto, 2013; BolsoniSilva \& Loureiro, 2015; Edmondson et al., 2007; Fitts, et al.; Segrin, 2000) uma vez que além de avaliar habilidades sociais, mensurou também consequências e sentimentos que são bons indicadores para a competência com que têm sido emitidas as habilidades sociais frente a diferentes interações sociais.

Por outro lado, os comportamentos não habilidosos que sugerem déficits e/ou excessos foram mais frequentes para o grupo clínico, bem como as percepções de consequências negativas e sentimentos negativos associados, o que se refletiu no escore de Dificuldades. As dificuldades foram a de se expressaram em diversas habilidades sociais avaliadas, tais como, apresentar seminários, falar em público, conversar, expressar sentimento positivo, expressar sentimento negativo, expressar opinião, fazer e receber críticas. Ainda que os estudos citados (Biasoto, 2013; Edmondson et al., 2007; Fitts et al., 2009; Segrin, 2000) não tenham avaliado todas essas variáveis simultaneamente e sim habilidades sociais de maneira geral, os achados da presente pesquisa permitem afirmar a relação inversa entre déficits de habilidades sociais e depressão.

Nesse sentido, os achados do estudo são concordantes com Segrin (2000) reforçando a afirmativa da relação inversa entre habilidades sociais e depressão. Como decorrência dessa constatação, pode-se pensar que possivelmente, quanto mais frequente e com qualidade for a emissão de comportamentos de habilidades sociais, maior será a probabilidade de solução de problemas e de respostas positivas aos eventos adversos que podem ocorrer na vida universitária. Foram considerados preditores de depressão o modo de lidar com interações sociais aversivas (Dozois \& Dobson, 2004), com estressores ambientais (Morrison-Valfre, 2009) e com experiências negativas da vida adulta (Morrison-Valfre, 2009). Assim, considera-se que a universidade, ao exigir novas demandas dos estudantes (estudo, convivência com desconhecidos, autoridades e distância com familiares, namorados e amigos), pode intensificar as interações sociais aversivas ou a convivência com estressores. Analisando-se os dados do estudo verificou-se que as habilidades sociais avaliadas que predisseram depressão, pela Análise de Regressão Logística, foram Potencialidades e Dificuldades (QHCUniversitários e Auto-afirmação na Expressão de Sentimento Positivo e Conversação e Desenvoltura Social (IHS-DelPrette). Tais achados estão em consonância com os estudos de Segrin (2000) e também com Edmondson et al. (2007) que verificaram a relação direta entre competência social e afeto, bem como com Fitts et al. (2009) que identificaram a associação do comportamento agressivo com depressão, o que no caso da presente pesquisa está relacionado com a categoria Dificuldades.

Como contribuição específica deste estudo, destacase a importância dos achados quanto a consequências e sentimentos percebidos pelos universitários, os quais apontam para a competência na emissão de comportamentos de habilidades sociais, de forma a resolver problemas e manter relacionamentos interpessoais positivos (consequências positivas), bem como sentir-se bem (sentimentos positivos). Tais dados reiteram a relevância de se considerar não apenas a frequência de habilidades sociais, mas também ater-se às consequências obtidas nas interações sociais, como assinalado por Libet e Lewinsohn (1973). Retomando ao se analisar as dificuldades e consequências negativas relacionadas à depressão faz-se necessário citar Marroquín (2011) e Evraire e Dozois (2011) que afirmaram que as pessoas com depressão acabam emitindo comportamentos que levam ao afastamento dos interlocutores, com consequências negativas, as quais, por sua vez, aumentam a probabilidade das pessoas com depressão buscarem o isolamento.

Em sendo a depressão na universidade um dos transtornos mais frequentes (Fang et al., 2010; Neves \& Dalgalarrondo, 2007) e com comorbidades (Dozois \& Dobson, 2004; Richards, 2011) considera-se que o repertório de habilidades sociais pode funcionar como um fator de proteção (Dozois \& Dobson, 2004) relevante para a depressão em universitários e também para o desenvolvimento de outras comorbidades. Sabe-se, então, que a presença de comorbidades é muito frequente (Johanson et al., 2013; Starr et al., 2014) e que tal condição tem graves implicações para a saúde e o prognóstico (Newby et al., 2013). De acordo com Aradilla-Herrero et al. (2014) universitários com depressão têm risco aumentado de suicídio e baixa autoestima assim, se os sintomas forem identificados precocemente e se os estudantes estiverem incluídos em programas de intervenções para ampliar suas 
habilidades sociais, tais riscos poderão ser minimizados, o que favorece mais saúde e desempenhos social e acadêmico.

Nesse sentido os dados do presente estudo, sobretudo porque controlou as variáveis sexo e escolaridade, contribuem com informações relevantes quanto a frequência e a qualidade de habilidades sociais, o que pode se útil em estudos futuros com foco na avaliação e intervenção com tal população. Outro ponto a ser destacado foi ter avaliado, além de múltiplos comportamentos de habilidades sociais, também comportamentos de agressividade e de inassertividade (comportamentos não habilidosos) o que em concordância com o estudo de Fitts, Sebby e Zlokovich (2009) ressalta a relevância de combinar, em uma mesma avaliação, recursos/ potencialidades e dificuldades.

\section{Considerações Finais}

As principais contribuições da presente pesquisa foram de avaliar múltiplos comportamentos de habilidades sociais, enfocando potencialidades, dificuldades e percepções de consequências para as interações. $\mathrm{O}$ controle rigoroso de variáveis para a composição dos grupos clínico e não clínico, o uso de instrumentos com qualidades psicométricas bem estabelecidas minimizou os viéses, colocando como foco de fato as variáveis de interesse, habilidades sociais e depressão. Constatou-se que o repertório deficitário de habilidades sociais foi preditor de depressão em universitários. Pesquisas futuras poderão abordar as comorbidades e o seu impacto para as habilidades sociais, buscando verificar se há especificidades nos repertórios, na dependência da presença de manifestações de psicopatologia associadas.

\section{Referências}

American Psychiatric Association. (2014). Diagnostic and statistical manual of mental disorders, fifth edition (DSM-V). Porto Alegre: Artmed Editora.

Andrade, L.H., Wang, Y. P., Andreoni, S., Silveira, C. M., Alexandrino-Silva, C., Siu, E. R., ... Viana, M. C. (2012). Mental disorders in megacities: findings from the São Paulo megacity mental health survey, Brazil. Plos-One, 7(2), 1-7. doi: 10.1371/journal.pone.0031879

Aradilla-Herrero, A., Tomás-Sábado, J., \& Gómez-Benito, J. (2014). Associations between emotional intelligence, depression and suicide risk in nursing students. Nurse Education Today, 34(4), 520-525.

Biasoto, F. F. (2013). Habilidades sociais e sofrimento psicológico. Arquivos Brasileiros de Psicologia, 65(1), 38-50. Retrieved from http://pepsic.bvsalud.org/scielo.php?script=sci_arttext\& pid $=$ S1809-52672013000100004

Bolsoni-Silva, A. T. \& Carrara, K. (2010). Habilidades sociais e análise do comportamento: Compatibilidades e dissensões conceitual-metodológicas. Psicologia em Revista (Online), 16(2), 330-350. http://dx.doi.org/10.5752/P.1678$9563.2010 \mathrm{v} 16 \mathrm{n} 2 \mathrm{p} 330$.
Bolsoni-Silva, A. T., \& Guerra, B. T. (2014). O impacto da depressão para as interações sociais de universitários. Estudos e Pesquisas em Psicologia, 14(2), 429-452. Retrieved from http://www.e-publicacoes.uerj.br/index.php/revispsi/article/ view/12649/9823

Bolsoni-Silva, A. T., \& Loureiro, S. R. (2015). Anxiety and depression in brazilian undergraduate students: the role of sociodemographic variables, undergraduate course characteristics and social skills. British Journal of Applied Science \& Technology, 5(3), 297-307. doi: 10.9734/ BJAST/2015/13004.

Costa, E. F. de O., Santana, Y.S., Santos, A.T.R. de A., Martins, A.N.; Melo, E.V. de, \& Andrade, T. M. (2012). Sintomas depressivos entre internos de medicina em uma universidade pública brasileira. Revista da Associação Médica Brasileira, $58(1), 53-$

Cunha, J. A. (2001). Manual da versão em português das Escalas Beck. São Paulo: Casa do Psicólogo.

Del Prette, A., \& Del Prette, Z. A. P. (2001). Inventário de habilidades sociais (IHS- Del Prette): Manual de aplicação, apuração e interpretação. São Paulo: Casa do Psicólogo.

Del Prette, Z. A. P., \& Del Prette, A. (2013). Social Skills Inventory (SSI-Del-Prette): Characteristics and studies in Brazil. In F. L. Osório (Org.), Social anxiety disorders: From theory to practice (pp. 49-62). Nova Iorque: Nova Science Publishers.

Del-Ben, C. M., Vilela, J. A. A., Crippa, J. A. S., Hallak, J. E. C., Labate, C. M., \& Zuardi, A. W. (2001). Confiabilidade da "Entrevista Clínica Estruturada para o DSM-IV - Versão Clínica" traduzida para o português. Revista Brasileira de Psiquiatria, 23(3), 156-159. doi: http://dx.doi.org/10.1590/ S1516-44462001000300008.

Dozois, J. A., \& Dobson, K. S. (2004). The prevention of anxiety and depression. Theory, research and practice. Washington, DC: American Psychological Association.

Edmondson, C. B., Conger, J. C., \& Conger, A. J. (2007). Social skills in college students with high trait anger. Journal of Social and Clinical Psychology, 26(5), 575-594. doi: http://dx.doi. org/10.1521/jscp.2007.26.5.575.

Evraire, L. E., \& Dozois, D. J. A. (2011). An integrative model of excessive reassurance seeking and negative feedback seeking in the development and maintenance of depression. Clinical Psychology Review, 31(8), 1291-1303. doi: http://dx.doi. org/10.1016/j.cpr.2011.07.014.

Fang, D., Young, C., Golshan, S., Fellows, I., Moutier, C., \& Zisook, S. (2010). Depression in Premedical Undergraduates: a CrossSectional Survey. Primary Care Companion Journal of Clinical Psychiatry, 12(6), e1-e6. doi: http://dx.doi.org/10.4088/ PCC.10m00958blu.

Fitts, S. D., Sebby, R. A., \& Zlokovich, M. S. (2009). Humor styles as mediators of the shyness-loneliness relationship. North American Journal of Psychology, 11(2), 257-272. Retrieved from http://www.freepatentsonline.com/article/NorthAmerican-Journal-Psychology/200919506.html

Johansson, R., Carlbring, P., Heedman, Å., Paxling B., \& Andersson. G. (2013). Depression, anxiety and their comorbidity in the Swedish general population: Point prevalence and the effect on health-related quality of life. Peer Review-Reviewed \& Open Access, 1, e98. doi: 10.7717/peerj.98 
Libet, J. M., \& Lewinsohn, P. M. (1973). Concept of social skill with special reference to the behavior of depressed persons. Journal of Consulting and Clinical Psychology, 40(2), 304-312. Retrieved from http://www.ncbi.nlm.nih.gov/ pubmed/21983267

Marroquín, B. (2011). Interpersonal emotion regulation as a mechanism of social support in depression. Clinical Psychology Review, 31(8), 1276-1290. doi: http://dx.doi. org/10.1016/j.cpr.2011.09.005.

Morrison-Valfre, M. (2009). Foundations of mental health care. Oregon: Mosby Elsevier.

Neves, M. C. C., \& Dalgalarrondo, P. (2007). Transtornos mentais auto-referidos em estudantes universitários. Jornal Brasileiro de Psiquiatria, 56(4), 237-244. http://dx.doi.org/10.1590/ S0047-20852007000400001.

Newby, J.M., Mackenzie, A., Williams, A. D.; McIntyre, K.; S. Watts, S.; Wong, N., \& Andrews, G. (2013). Internet cognitive behavioural therapy for mixed anxiety and depression: a randomized controlled trial and evidence of effectiveness in primary care. Psychological Medicine, 43, 2635-2648. doi:10.1017/S0033291713000111
Osada, J., Rojas, M., Rosales, C., \& Veja-Dienstmaier, J. M. (2010). Sintomatología ansiosa y depresiva em studiantes de medicina. Revista de Neuropsiquiatria, 77(1), 15-19. doi: 10.20453/rnp. v73i1.1651.

Razzouk, D., Alvarez, C. E., \& Mari, J. J. (2009). O impacto econômico e o custo social da depressão. In A. L. T. Lacerda, L. C. Quarantini, J. A. Del Porto, \& A. M. A. Miranda-Scippa (Eds.), Depressão: Do neurônio ao funcionamento social (pp. 85-98). Porto Alegre: Artmed.

Richards, D. (2011). Prevalence and clinical course of depression: A review. Clinical Psychology Review, 31(7), 1117-1125. doi: http://dx.doi.org/10.1016/j.cpr.2011.07.004.

Segrin, C. (2000). Social skills deficits associated with depression. Clinical Psychology Review, 20(3), 379-403. doi: http://dx.doi. org/10.1016/S0272-7358(98)00104-4.

Shamsuddin, K., Fadzil, F., Ismail, W. S. W., Shamsul, S. A., Omar, K., Noor Jaffar, N. A. M. A., ... Raynuha, M. (2013). Correlates of depression, anxiety and stress among Malaysian university students. Asian Journal of Psychiatry, 6(4), 318-323. doi: 10.1016/j.ajp.2013.01.014.

Starr, L. R., Hammen, C.; Connolly, N. P., \& Brennan, P. A. (2014). Does relational dysfunction mediate the association between anxiety disorders and later depression? Testing an interpersonal model of comorbidity. Depression and Anxiety, 31, 77-86. doi: 10.1002/da.22172. 